\title{
Focused Review on Transthoracic Echocardiographic Assessment of Patients with Continuous Axial Left Ventricular Assist Devices
}

\author{
Yan Topilsky, ${ }^{1}$ Simon Maltais, ${ }^{2}$ Jae K. Oh, ${ }^{1}$ Fawn W. Atchison, ${ }^{3}$ Louis P. Perrault, ${ }^{4}$ \\ Michel Carrier, ${ }^{4}$ and Soon J. Park ${ }^{2}$ \\ ${ }^{1}$ Division of Cardiology, Mayo Clinic College of Medicine, Rochester, MN 55905, USA \\ ${ }^{2}$ Division of Cardiovascular Surgery, Mayo Clinic College of Medicine, 200 First Street S. W. Rochester, MN 55905, USA \\ ${ }^{3}$ Department of Anesthesiology, Mayo Clinic College of Medicine, Rochester, MN 55905, USA \\ ${ }^{4}$ Department of Cardiac Surgery, Montreal Heart Institute, Montreal, QC, Canada H1T1C8 \\ Correspondence should be addressed to Soon J. Park, park.soon@mayo.edu
}

Received 2 November 2010; Accepted 17 December 2010

Academic Editor: Dirk Westermann

Copyright $\odot 2011$ Yan Topilsky et al. This is an open access article distributed under the Creative Commons Attribution License, which permits unrestricted use, distribution, and reproduction in any medium, provided the original work is properly cited.

Left ventricular assist devices (LVADs) are systems for mechanical support for patients with end-stage heart failure. Preoperative, postoperative and comprehensive followup with transthoracic echocardiography has a major role in LVAD patient management. In this paper, we will present briefly the hemodynamics of axial-flow LVAD, the rationale, and available data for a complete and organized echocardiographic assessment in these patients including preoperative assessment, postoperative and long-term evaluation.

\section{Introduction}

Left ventricular assist devices (LVADs) are systems for mechanical support for patients with end-stage heart failure. They are effective in supporting the circulation for weeks to years as a bridge to transplant (BTT) or destination therapy (DT) [1-4]. The newer axial-flow devices have been designed in an effort to minimize operative risk, improve durability, and lower the risk of device-related adverse events by reducing the number of moving parts in the device and device size [5-7]. Continuous axial flow LVAD can provide effective hemodynamic support for prolonged periods, improving functional status and quality of life $[8,9]$. The designation axial flow device refers specifically to the design and shape of the impeller, and therefore the route by which fluid is accelerated. The impellers of axial flow pumps generally add energy by deflecting flow in the circumferential direction. Despite their small size, these pumps can provide flows of up to $8-10 \mathrm{~L} / \mathrm{min}$, sufficient to support a large adult patient. Currently, there are two major types of axial pumps in the USA: Heartmate II (Heartmate II, Thoratec, Pleasanton, CA) and Jarvic 2000 (Jarvik, New York City, New York, USA). The Jarvik 2000 is an intraventricular axial flow pump that measures $25 \mathrm{~mm}$ in diameter by $55 \mathrm{~mm}$ in length with a weight of approximately $85 \mathrm{~g}$. It consists of the pump positioned inside the left ventricular cavity and the outflow cannula which can be anastomosed to the right anterior aspect of the ascending aorta or to the descending aorta. The impeller rotates in the left ventricular cavity at speeds of 8,000 to 12,000 revolutions per minute (RPM) to deliver 2 to $7 \mathrm{~L} / \mathrm{min}$. The pulse control circuit allows the user to adjust the rotational speed of the pump manually.

The HeartMate II LVAD (Thoratec, Pleasanton, CA) is a continuous, axial-flow LVAD, positioned in succession to the left ventricle (LV). It consists of a spinning rotor pump as its lone moving part, an inflow cannula, an outflow cannula, and a single driveline that exits percutaneously towards the electronic controller $[6,7]$. The inflow cannula is inserted into the apex of the $\mathrm{LV}$, and the outflow cannula is anastomosed to the right anterior aspect of the ascending aorta. The LVAD pump is placed within the preperitoneal space. In both pumps, the percutaneous lead carries the electrical cable to an electronic controler and battery packs (one in the Jarvik and two in the HeartMate II), which are worn on a belt and a shoulder holster, respectively. The spinning rotor draws blood from the inflow 
cannula throughout cardiac diastole and systole, propelling it into the aorta. Preoperative, postoperative, and followup transthoracic echocardiography (TTE) has a major role in LVAD patient management. In this paper, we will present briefly the hemodynamics of axial-flow LVAD, focusing on the HeartMate II, and the rationale and available data for a comprehensive echocardiographic assessment in these patients including preoperative assessment, postoperative and long-term evaluation.

LVAD Performance Parameters. The system-provided parameters of speed, power, PI, and estimated flow for the HeartMate II serve as important indicators of proper LVAD function. It is important to view each of these device parameters in the context of the patient's overall condition. Once baseline values representing a good level of patient support are established, the degree of change in a parameter usually has more clinical significance than its absolute value. Briefly, the pump speed will be determined during a speed ramp study. Pump power is a direct measurement of motor voltage and current. Increases in pump speed, flow, or physiological demand will increase pump power. Specifically, gradual power increases, power values greater than 10-12 watts, or abrupt changes in power should raise concern for possible thrombus inside the pump. When the LV contracts, the increase in ventricular pressure causes an increase in pump flow during systole. The magnitude of these flow changes is measured to produce the PI. The PI represents cardiac pulsatility and is related to the magnitude of assistance provided by the LVAD. Higher values indicate more ventricular filling or better contractility (pump is providing less support to the $\mathrm{LV}$ ), while lower values indicate less ventricular filling or lower contractility. Pump flow is estimated based on power. Since it is a calculated value, it becomes imprecise at low and high regions of the powerflow relationship. Therefore, any increase in power not related to an increased flow, such as thrombus, will cause an erroneously high flow. Conversely, an occlusion of flow path (inflow obstruction due to malposition or suction events) will decrease power and calculated flow. In either situation, an independent assessment of pump output using the TTE should be performed.

\section{Evaluation of Axial-Flow LVAD Hemodynamics}

2.1. The "Tight Relation" between the LV, the Left Atrium, and the Aorta. Axial-flow pumps are connected in series to the LV by the inflow cannula, with the LV being the direct source of preload essential for LVAD output. They continuously unload the LV during the entire cardiac cycle, eliminating the isometric contraction and relaxation phases of normal cardiac activity. Although considered to be continuous flow assist devices, working in succession to the LV, the true hemodynamic profile depends on the pump speed, the LV contractility, the preload, and the afterload conditions.

The volume of flow generated by continuous flow LVAD is determined by the speed of the rotation of the pump and by the differential pressure that exists across the device. For a specified speed, flow varies inversely with the pressure difference [10]. The LVAD is connected to the circulation by the inflow conduit on the LV apex, while the outflow graft is secured to the aorta. With these connections, throughout the cardiac cycle, the pump differential pressure is equal to the aortic pressure minus the LV pressure. In other words, the flow increases with increasing LV pressure (LVAD preload), or decreasing aortic pressure (LVAD afterload). The dynamic parameter that determines the pump's differential pressure is the LV pressure, which in turn is dependent on its contractile reserve. Even a severely depressed LV will have the possibility to generate some residual rhythmic contraction that will create pulse pressure. This pressure fluctuation at the pump inflow will change the pump differential pressure, which in turn will alter the pump flow. Therefore, any residual contraction of the LV will be transduced as a flow pulse delivered to the aorta, creating, under most circumstances, pulsatile systemic flow.

At maximal speed, the LV end-diastolic volume decreases to a minimum, due to maximal decompression by the LVAD. This is comparable to a state of "hypovolemic shock." The reduced preload is translated to subphysiological LV and left atrial systolic and diastolic pressures. Furthermore, the already failing LV exposed to reduced preload decreases its contractility according to Starling's response. This minimizes the increase in LV systolic pressure, resulting in a nonsignificant systolic increase in LVAD preload and output. The systemic circulation is continuously supported by the maximal LVAD output, preventing significant changes in the aortic pressure and flow between systole and diastole. LV systolic pressure does not increase above aortic pressures and is insufficient to allow aortic ejection. The LV remains the direct source of LVAD preload but does not contribute independently to the cardiac output.

As pump flow decreases to submaximal values $(10,000$ to $11,000 \mathrm{rpm}$ ), pulsatility is generated. More blood is left in the ventricle, enhancing LV contractility as LV systolic pressure rises. The increased LV systolic pressure translates into increased systolic LVAD preload generating pulsatility. Under submaximal LVAD speed, systolic LV pressure does not increase above aortic pressure and is still insufficient to allow aortic ejection (aortic valve opening). The LV still does not contribute independently to cardiac output.

Reducing pump speed further increases the amount of blood left in the ventricle. LV contractility increases through the Starling's response, and the LV systolic pressure increases above aortic pressure allowing aortic valve opening and systolic ejection. Aortic valve closure (and opening) can therefore be recognized by the reappearance of the normal dicrotic notch in the aortic pressure curve.

\subsection{Hemodynamic Impact of the LVAD on Right-Sided Cham-} bers. As previously discussed, the axial-flow pumps are directly connected to the LV through the inflow cannula, making the LV the direct source of preload. The LV, in turn, receives preload from the right ventricle (RV) through the pulmonary circulation. This important concept is central to optimal pump function. Right heart dysfunction (RHD) is 
a frequent concern after LVAD implantation, as it occurs in one-third of patients. It contributes significantly to postoperative morbidity and mortality [11]. The LVAD mechanically unloads the LV, resulting in subphysiological left atrial and left ventricular pressures. On the other hand, right ventricular and right atrial pressures may be elevated after LVAD implantation [5]. Excessive unloading of left heart chambers may result in a complex biphasic effect on RV function. Following LVAD implantation, there is a significant decrease in mean left atrial and mean pulmonary pressures [12]. The reduced RV afterload may therefore improve its function and total output. However, bowing of the interventricular septum away from the right ventricle into the decompressed LV may reduce the efficiency of RV contraction by destabilizing the hinge upon which the RV contracts [11, 12]. Moreover, the $\mathrm{RV}$ may receive excessive venous return owing to the LVAD effective forward flow through the systemic circulation, resulting in RV dilatation and dysfunction [13]. Maintaining the septum in the midline position requires maintenance of adequate or appropriate $\mathrm{LV}$ volume. This can result in suboptimal LVAD flow, which prevents overcirculation that could overwhelm the functional capacity of the RV. If cardiac output decreases because of lack of LV filling due to RV failure, high pulmonary resistance, or significant tricuspid regurgitation (TR), it is hazardous to attempt to improve the patient's condition by increasing pump speed. Without improvement in left ventricular inflow, increasing the speed will cause a further decrease in the size of the LV cavity compounding the leftward septal shift. This further impairs RV function and increases TR severity, decreasing the already compromised LV inflow. This worsening spiral, sometimes referred to as the "suction cascade," may cause the septum to encroach upon the inflow cannula, increasing inflow velocities, but decreasing the LVAD preload. This requires immediate intervention to avoid a vicious cycle that can eventually lead to a fatal outcome.

\section{3. "Step by Step" Preoperative Role of Echocardiography in LVAD Patients}

Transthoracic echocardiographic assessment of patient undergoing VAD insertion involves aspects related both to general echocardiographic examinations and to specific considerations associated with the LVAD. We will concentrate on specific imaging concerns pertaining to LVAD patients. For simplicity purposes, we will divide this section in three distinct parts: (1) preoperative evaluation; (2) postoperative assessment; (3) long-term echocardiographic considerations.

\subsection{Preoperative Surgical Evaluation. Pre-LVAD insertion} examination of the heart and large vessels is done for two main purposes: (1) evaluating suitability of patient for LVAD placement; (2) assessing significant cardiac abnormalities that could lead to postoperative complications. Important preoperative imaging include the evaluation of left heart chamber function and structure, the quantification of RV function and TR, and the assessment for aortic and mitral regurgitation. Other specific LVAD concerns are the presence of a patent foramen ovale, or the presence of intracardiac clots. These are routinely evaluated with transesophageal echocardiography in the operating room.

\subsubsection{Preoperative Evaluation of the Left Heart Chambers.} The evaluation of the LV function before LVAD implantation will most commonly show depressed function with either a dilated or normal sized ventricle, depending on the cause of heart failure. The LV ejection fraction (LVEF) preLVAD insertion is typically $<25 \%-30 \%$. Significant diastolic dysfunction is also usually present. The presence of a restrictive LV diastolic physiology reflects increased LV and LA pressures and, when severe, supports the indication of LVAD implantation [14]. Another common finding in patients eligible for LVAD implantation is significant functional mitral regurgitation, due to mitral annulus dilatation, and apical tethering of mitral leaflets secondary to the geometrical changes imposed on the left ventricle [15].

3.1.2. Right Ventricular Function and TR. The central role of $\mathrm{RV}$ function has already been discussed in detail emphasizing the importance of a proper functional evaluation of the RV before LVAD implantation. The RV is a complex structure and is incompletely visualized in any single $2 \mathrm{D}$ echocardiographic view. Two methods are commonly used to evaluate RV function before LVAD implantation. The first is semiquantitative assessment of RV function and dilatation, using the four chambers and inflow views. This assessment is based on visual appreciation of longitudinal and radial RV motion. A more quantitative approach was recently proposed in recent reports; the global RV fractional area change is calculated as the RV fractional area change $=(\mathrm{RV}$ diastolic area-RV systolic area)/RV diastolic area, with $\mathrm{RV}$ diastolic and systolic areas traced in the 4 chamber views [16]. An RV fractional area change (RVFAC) of $40 \%$ or higher is normal. Typically, the RVFAC in a patient needing an LVAD implantation is $20 \%$ to $30 \%$. Patients with an RVFAC $<20 \%$ are more prone to postoperative RV failure. For estimation of TR, a combination of qualitative and quantitative methods are used as described previously [17]. More than moderate TR requires surgical correction either through a TV annuloplasty or a tricuspid valve replacement [18].

3.1.3. Aortic Regurgitation. Diagnosis of significant pre- and postoperative aortic regurgitation (AR) is crucial in patients receiving an LVAD. The LVAD draws blood from the LV and ejects it into the aorta creating subphysiological LV pressures. The retrograde aorta to LV gradient increases and continues throughout the cardiac cycle, including most of the systolic phase. During maximal LVAD output, the aortic valve is permanently closed, encountering this gradient constantly. The combination of increased pressure gradient and exposure time results in increased regurgitant volume after LVAD insertion. The regurgitant volume increases LVAD preload and LV dimensions and causes secondary pump flow volume upregulation. This in turn results in further increase in blood ejection to the ascending aorta. 


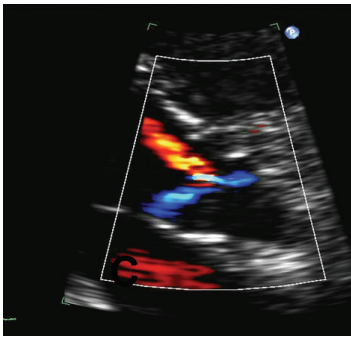

(a)

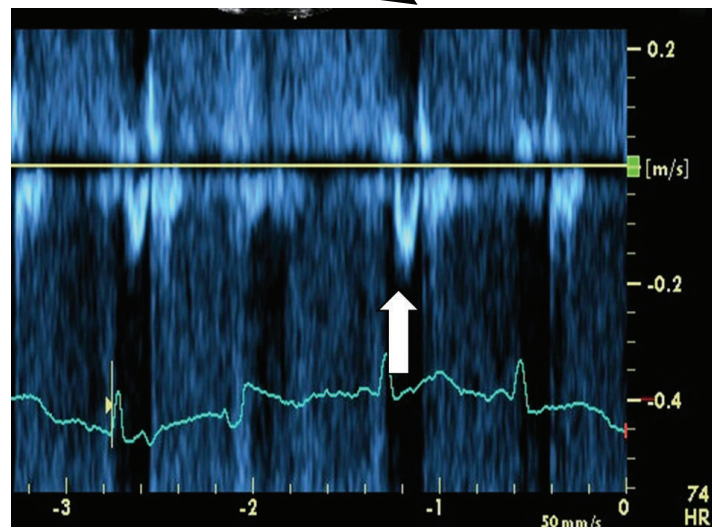

(f)

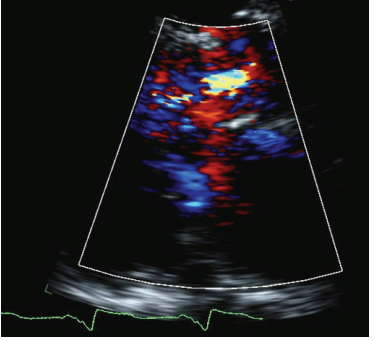

(b)

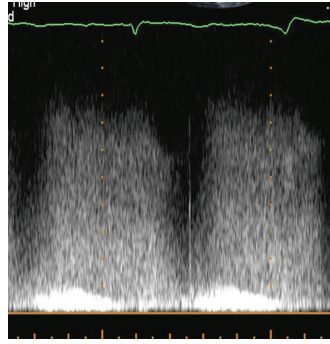

(c)

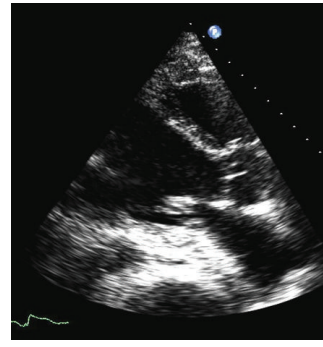

(d)
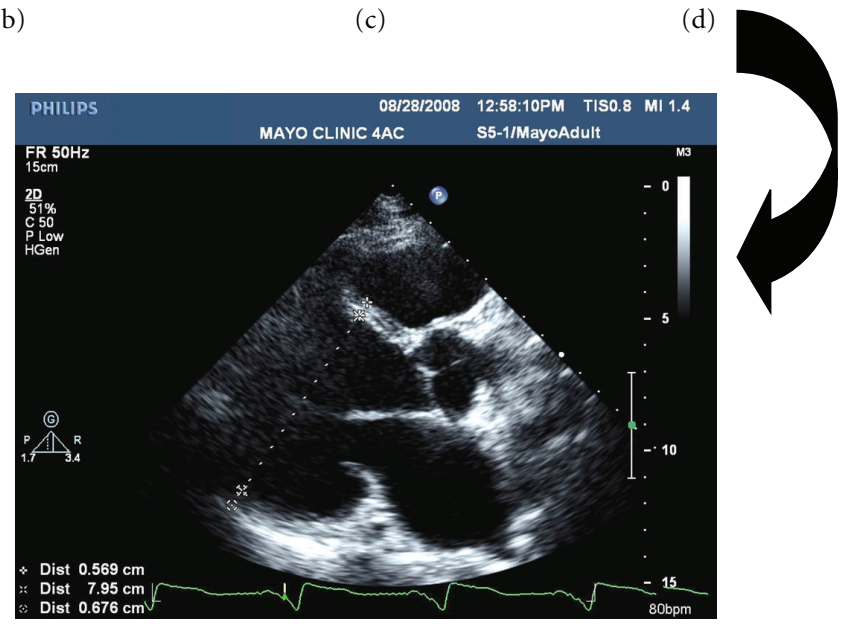

(e)

FIGURE 1: Aortic regurgitation after implantation. Patient presented with new onset heart failure 6 months after LVAD implantation. LVAD evaluation showed increased power and LVAD flow. (a) Pre-LVAD parasternal long axis view showing mild diastolic aortic regurgitation. (b), (c), and (d) Immediately after LVAD, blood is pumped from the LV into the aorta creating subphysiological LV pressures. The retrograde aorta to LV gradient increases and continues throughout the cardiac cycle, including most of the systolic phase. Aortic regurgitation has increased in volume and takes place during most of the cardiac cycle. (e) and (f) Six months after LVAD surgery. Aortic regurgitation deteriorated secondary to closed aortic valve encountering high retrograde pressure gradient throughout the cardiac cycle. The progressing regurgitant volume increased left ventricular diameter, which in turn amplified LVAD preload and output. Pump output spiraled up to very high levels, while actual systemic blood flow fell. The end result was a "futile cycle" consisting of high pump flow, low total cardiac output, and high left ventricular and left atrial pressures.

Pump output spirals up to very high levels, while actual systemic blood flow falls. The increased LV dimensions may result in apical tethering of mitral leaflets and functional MR. The end result is a "futile cycle" consisting of high pump flow, low total cardiac output, and high LV and LA pressures (Figure 1). In our institution, patients with significant AR as a result of structural problems of the valve undergo aortic valve replacement or surgical closure of the aortic valve leaflets (Park's stitch) [19].

3.1.4. Patent Foramen Ovale (PFO). Investigation of a Patent Foramen Ovale (PFO) should always be performed before implantation of an LVAD. Because a PFO is common (around 25\% of the population), meticulous care should be taken to identify its presence. We find that the best way to identify a PFO by TTE is with the use of contrast (agitated saline or bubble study) concurrent with color Doppler. We usually perform the exam in the apical four-chamber view or the subcostal view focusing on the interatrial septum. Patients are taught to perform standard Valsalva maneuver before their echocardiographic examination: first, sustained straining against a closed epiglottis causing abdominal distension for 10 seconds before sudden release of the strain by deep inspiration. Adequate performance of Valsalva maneuver can be detected by a decrease in the left atrial and ventricular sizes with interatrial septum bulging to the left atrium. For contrast injection, we use an 18 French catheter inserted at the right antecubital vein, which is connected by an extension tube to a 3-way stopcock with two $10 \mathrm{~mL}$ Luer Lock syringes. One $\mathrm{mL}$ of patient's blood is drawn from the vein into a syringe containing $8 \mathrm{~mL}$ of sterile normal saline solution and $1 \mathrm{~mL}$ of air. The content is forcefully injected back and forth for few times between the two syringes to become a cloudy and foamy pink emulsion and then rapidly administered intravenously to the patient at baseline, before the Valsalva maneuver. PreLVAD implantation LA pressure surpasses the RA pressure. Because of this factor, investigation of a PFO with color Doppler echocardiography may show a left-to-right shunt, and a bubble study may not reveal a PFO due to the difficulty in producing a transient reversal of the left-to-right 


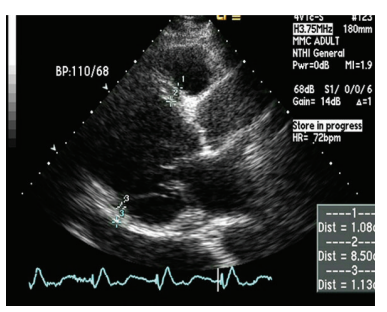

(a)

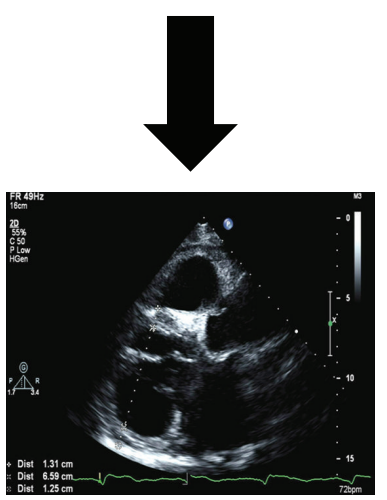

(e)

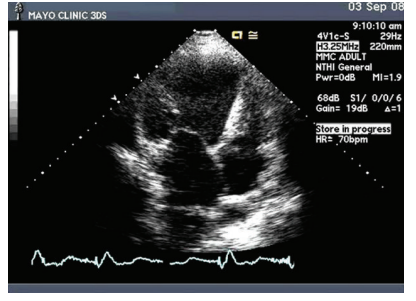

(b)
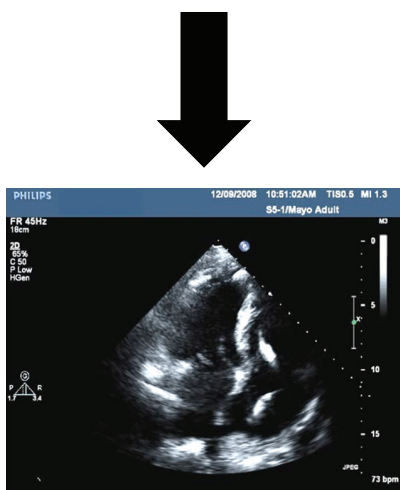

(f)

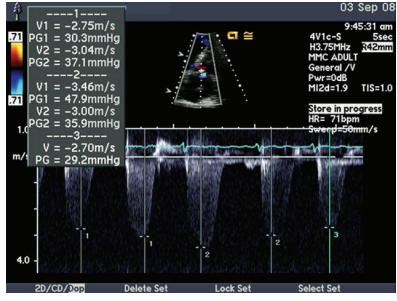

(c)
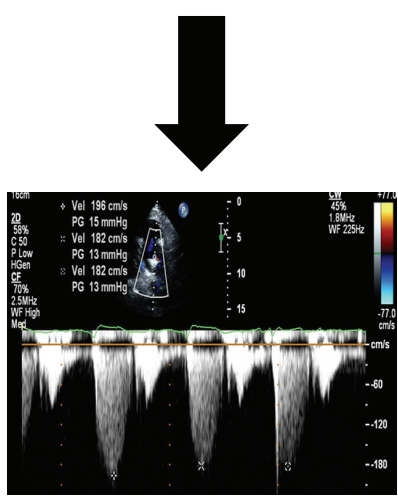

(g)

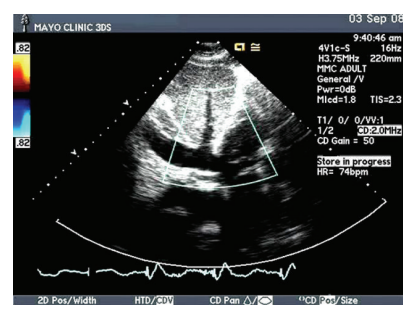

(d)
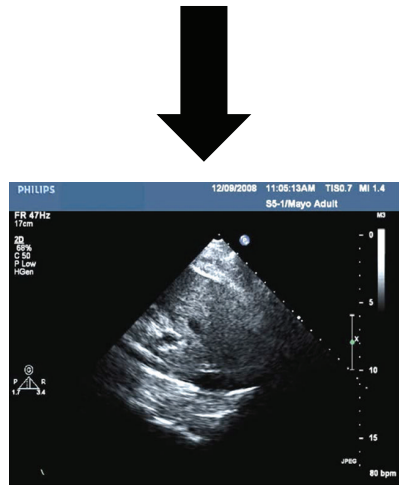

(h)

FIGURE 2: Interventricular dependency. Parasternal long axis view showing severely dilated LV (end-diastolic dimension 85 mm). (b) Apical 4-chamber view showing interventricular, interatrial septa shifted to the right, and increased tenting and annular dimensions of mitral valve apparatus. (c) and (d) Systolic right ventricular and right atrial pressure is increased. The same patient two months after surgery. (e) Enddiastolic diameter decreased, and septal and posterior walls have thickened. (f) Interventricular and interatrial septa are now shifted to the left. Mitral annulus and tenting area have decreased as well as functional mitral regurgitation. (g) and (h) Systolic right ventricular and right atrial pressures have decreased significantly.

pressure gradient in the presence of left heart failure, even when a Valsalva maneuver release is correctly applied. After insertion of an LVAD, there is LV unloading with decrease of the LA pressure. This hemodynamic change, in association with maintained or increased right heart pressures, may uncover the existence of the PFO, usually with the use of intraoperative TEE. Those hemodynamic conditions can also favor a paradoxical embolism which may result in stroke or even pump thrombosis. One of the serious and more common consequences of this sequence of events is the development of severe hypoxemia due to the significant right-to-left shunt stressing the need to evaluate for PFO before and after LVAD implantation.

\section{Postoperative Value of Echocardiography in LVAD Patients}

Postsurgical TTE evaluation is performed for two main purposes: (1) to evaluate the surgical results of the LVAD implantation; (2) to determine reasons for postoperative hemodynamic compromises. Specifically, important routine postoperative imaging concerns include the following: (1) overall structure and function of left heart chambers; (2) quantification of RV function and TR; (3) proper inflow and outflow cannula placement.

4.1. Postoperative Evaluation of Left Heart Chambers. After LVAD insertion, the LV and the LA are unloaded with a reduction in their size [20]. Neutral or slight leftward interventricular and interatrial septa position indicates adequate LV and LA decompression (Figure 2). This appearance should persist throughout the subsequent examinations. If the LV is not decompressed after LVAD implantation, a rightward septum shift can be seen and suspicion of insufficient device ejection, or cannula obstruction should be immediately raised. In contrast, extreme leftward septal shift may indicate excessive decompression due to high pump RPM, significant tricuspid regurgitation, or RV dysfunction. Because LVAD promotes nonphysiological LV unloading, common parameters for LV function assessment such as LVEF become invalid.

4.2. Right Ventricular Function and TR. Up to one-third of patients will present with variable degrees of RV dysfunction following surgery [11]. This stresses the importance of a thorough re-examination of RV function and TR severity after LVAD insertion. The postoperative examination should follow the same protocol described in the pre-LVAD examination section. Once identified echocardiographically, TR severity should be assessed during pump flow adjustments. Such adjustments lead not only to the reduction of the TR but can also improve RV function. As part of the integrated estimation of right heart function, mean pulmonary pressure should be estimated using the mean of peak systolic tricuspid regurgitation velocity in end expiration. The right atrial pressure (RAP) should be estimated by the inferior vena cava diameter and its response to inspiration as described [21]. 
4.3. Assessment of Aortic and Mitral Regurgitation Severity. Estimation of AR severity should be part of every TTE evaluation as it may deteriorate secondary to the closed aortic valve encountering high retrograde pressure gradient, continued throughout the cardiac cycle. The most commonly used methods are visual estimation by color Doppler, the ratio of $A R$ jet area to the short axis area of the LVOT at the level of the aortic annulus, and the width of the regurgitant jet at its origin relative to the dimension of the LVOT in the parasternal long axis view. For mitral regurgitation (MR), in a normally functioning LVAD system, functional $\mathrm{MR}$ is expected to decrease significantly. When MR persists, a thorough evaluation of its cause should be performed. In our experience, functional MR "begets" functional MR just as in patients without LVAD. Suboptimal LVAD RPM setting results in increased LV dimension, mitral valve tenting, significant mitral regurgitation, and volume overload of left ventricle, culminating in increasing LV diameter and deteriorating functional MR (Figure 3). Whenever significant functional MR is encountered, a trial of increasing RPM under echocardiographic guidance should be tried.

4.4. Inflow Cannula Evaluation. The inflow cannula and its orientation within the left ventricular apex should be visualised on the four- and two-chamber views. The cannula should be aligned with the LV inflow tract. Color Doppler is an important component of the examination. A properly aligned inflow cannula should have a laminar and unidirectional flow from the ventricle to the device. Abnormally high velocity or turbulent flow suggests obstruction of the inflow cannula. The most common reasons for obstruction to flow in the inflow cannula are thrombus or intermittent obstruction of the cannula by the ventricular wall (Figure 4) [22]. Doppler assessment of the inflow cannula should be done in the four- and two-chamber views, as they are aligned with the central axis of a properly positioned inflow cannula. Pulsed Doppler assessment should show laminar, low velocity flow, with no regurgitation. Continuous Doppler is used for measurement of the maximal velocity along the inflow pathway from the ventricle to the LVAD. Particular attention should be paid to high velocities produced by cannula obstruction, and regurgitant flow suggestive of pump malfunction. Axial-flow devices such as the HeartMate II will normally show a pulsatile inflow pattern because the pump inflow originates from the beating $\mathrm{LV}$, resulting in periodic changes in flow throughout the cardiac cycle, reaching a maximum during systole, and minimum during diastole. This pattern is present even when the aortic valve does not open. Axial-flow devices show peak filling velocity between 0.7 and $2.0 \mathrm{~m} / \mathrm{s}$ according to preload and the remaining pumping action of the patient's heart.

4.5. Outflow Cannula. Interrogation of the outflow cannula by TTE is technically challenging. We advocate the use of (1) high left parasternal long axis view, which shows the end-toside anastomosis of the outflow cannula to the midascending aorta; (2) right parasternal view, with the patient lying on his right side, which shows the long axis of the outflow cannula traversing from the pump towards the right aspect of the ascending aorta. Color flow, PW, and CW Doppler are used to evaluate flow patterns of the outflow cannula. To measure flow velocity in the outflow graft, the PW sample volume should be at least $1 \mathrm{~cm}$ proximal to the aortic anastomosis. The peak velocity in the outflow graft in axial flow pumps usually ranges from 0.5 to $2.0 \mathrm{~m} / \mathrm{s}$, with unidirectional and slightly pulsatile flow [23], dependent on LVAD output and speed. Flow patterns of the aortic outflow cannula are significantly affected by the angle of insertion of the LVAD outflow cannula into the native aorta [24]. Connecting the LVAD outflow conduit at a shallower angle to the proximal aorta produces fewer secondary flows, lower shear stress on the aortic wall, and lower peak velocities. Sometimes velocity can be measured in the right parasternal view, with the flow directed towards the transducer.

\section{Evaluation of Postoperative Hemodynamic Instability}

The most common reasons for hemodynamic instability during the first postoperative days are hypovolemia (from intractable bleeding), acute RV dysfunction, cardiac tamponade, pulmonary emboli and LVAD dysfunction, most commonly secondary to impeller thrombosis [25].

Acute RV dysfunction can manifest itself in the previously described "suction cascade," including dilated hypocontractile RV, significant functional TR, small LV, and intermittent inflow cannula obstruction by the collapsed LV. Pulmonary embolism can cause acute RV dysfunction, will present in a similar way, and should be considered whenever right-sided pressures are higher than expected. Cardiac tamponade is sometimes very difficult to diagnose. Blood collections may be loculated and confined to a small area, compressing a particular chamber. Right or left atrial tamponade can occur with very small collections of blood. Right ventricular tamponade may be the consequence of a loculated substernal thrombus [25]. The unusual physiology of the LV makes the standard Doppler assessments for tamponade very challenging, since the device's echogenicity impairs thrombi visualization. On the other hand, LVAD dysfunction or thrombosis should be suspected with the following combination of findings: (1) rightward deviation of the interventricular and interatrial septum as a sign of deficient unloading of the LV and left atrium; (2) significant functional MR, due to insufficient LV unloading, mitral annular dilatation, and apical tethering of the mitral leaflets; (3) aortic valve opening every cardiac cycle due to increased LV systolic pressure; (4) decreased LVAD flow; (5) disturbed LVAD parameters, mainly increased power intake; (6) laboratory clues suggesting intravascular hemolysis (increased LDH, plasma hemoglobin, and bilirubin with decreased haptoglobin). This will be demonstrated by color, or PW Doppler evaluation of the cannula. Whenever the pump's impeller does not rotate, the LVAD system operates as a conduit connecting the ascending aorta to the left ventricular apex. Diastolic aortic pressure is higher than left ventricular diastolic pressure, and under conditions of impaired pump rotation, the pressure difference reverses the flow from the 


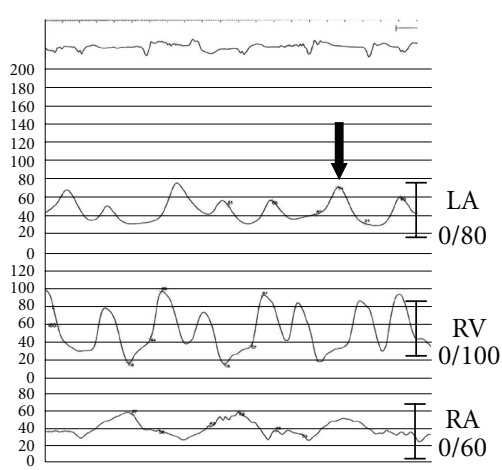

(a)

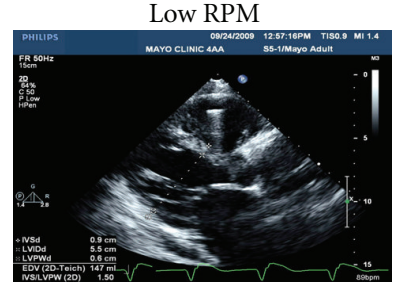

(b)

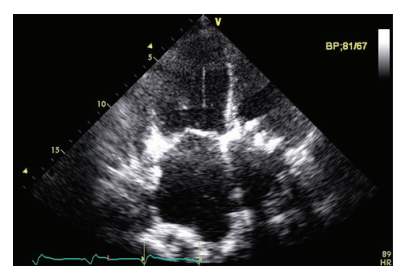

(c)

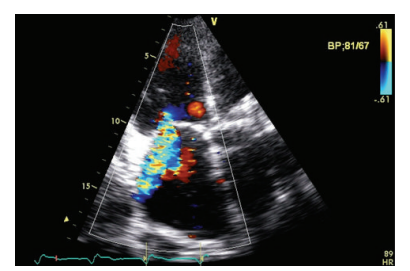

(d)

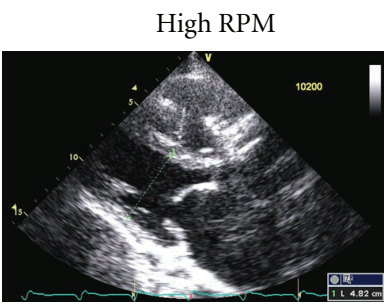

(e)

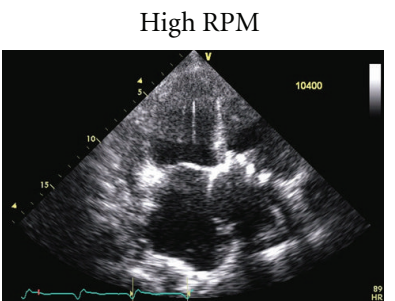

(f)

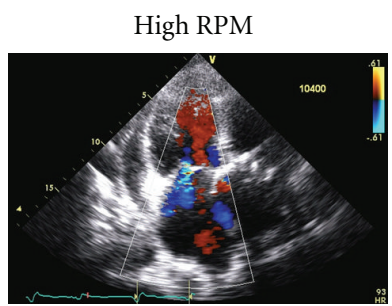

(g)

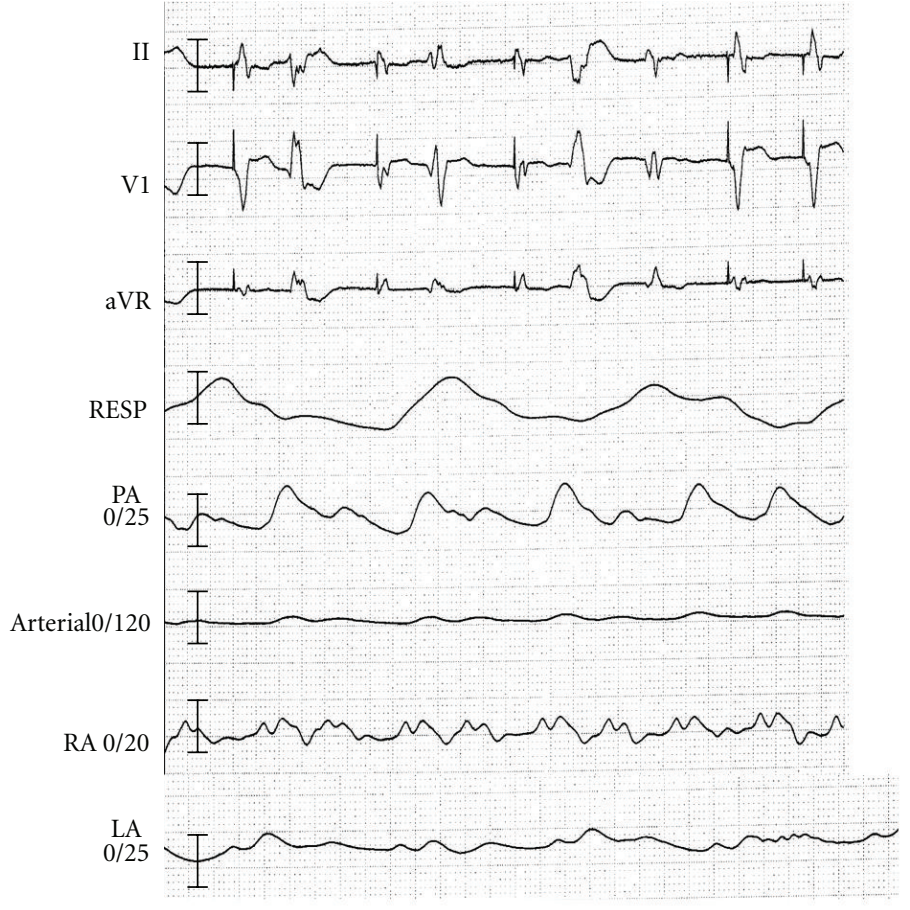

(h)

FIGURE 3: Severe functional mitral regurgitation. A patient presenting 17 months after LVAD implantation with failure to thrive and advanced right- and left-sided heart failure. (a) Hemodynamic right heart catheterization revealed markedly increased wedge and right atrial and ventricular pressures. Left atrial $\mathrm{V}$ wave (black arrow) reaching up to $70 \mathrm{mmHg}$ was recorded suggesting significant functional mitral regurgitation. Echocardiographic examination during baseline RPM settings showed increased LV diameter (a), interventricular, interatrial septa shifted to the right, increased mitral annular diameter and tenting diameters (b), and severe functional mitral regurgitation. Left ventricular assist device speed was increased under echocardiographic guidance. Left ventricular middiameter decreased in size (e). Interventricular, interatrial septa shifted to the left and mitral annular and tenting diameters decreased in size (f). Functional mitral regurgitation severity decreased significantly $(\mathrm{g})$. Right heart catheterization in the end of echocardiographic examination showed normal wedge, right ventricle, and right atrial pressures, and no $\mathrm{V}$ waves (h). Notably, cardiac output increased from 5.7 to 7.2 liters/minute after LVAD optimization.

ascending aorta through the outflow and inflow cannula and into the LV apex.

\section{Long-Term Echocardiographic Considerations in Patients with LVAD}

TTE is used in patients on chronic LVAD therapy for two main purposes: (1) routine LVAD optimization; (2) assessment of clinical deterioration or abnormal LVAD parameters associated with LVAD dysfunction.

6.1. Optimal LVAD Settings. The goal of LVAD implantation is to increase cardiac output while decreasing filling pressures. Optimal axial LVAD settings are still controversial. While some authors believe that maximal cardiac output and left chamber unloading are ideal, others are concerned by the long-term effects of LVAD working at maximal output [5]. 

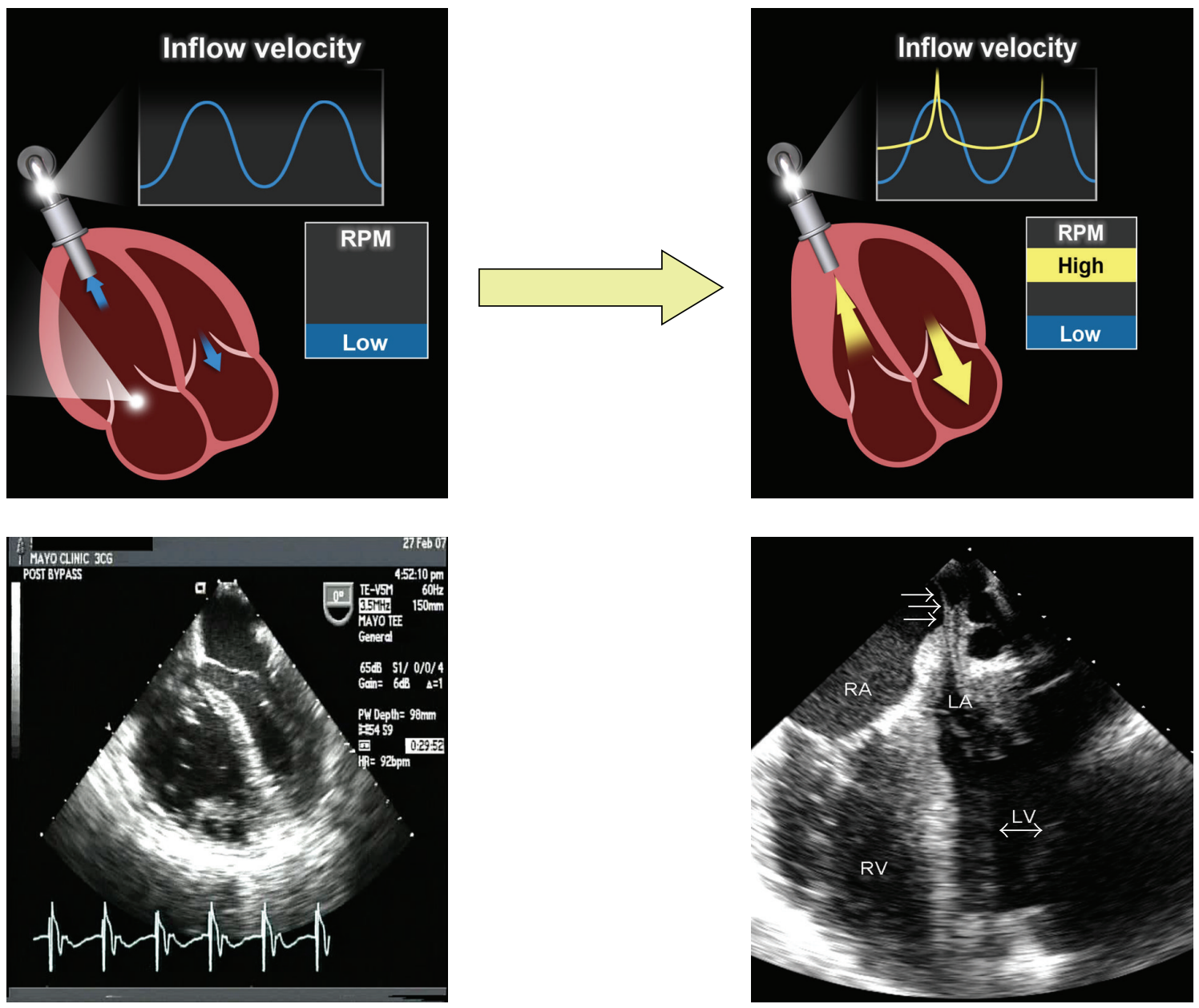

FIGURE 4: Inflow cannula obstruction, the "suction cascade". Four-chamber transthoracic view representing the classic "suction cascade" that can manifest itself by a dilated hypocontractile RV, significant functional TR, small left ventricle, and intermittent inflow cannula obstruction by the collapsed LV.

Possible complications of maximal LVAD output include increased prevalence of "suction events", hemolysis, stasis of blood proximal to the outflow cannula, and end-organ injury related to nonphysiologic continuous circulation. The clinical implications of continuous blood flow are currently unknown and have been a matter of significant debate. Another possible concern is stasis of blood proximal to the outflow cannula and ascending aorta anastomosis, when there is no forward flow through the aortic valve. This stagnant column of blood may theoretically result in thrombosis and embolism to coronaries or carotid arteries. Although this was described in the Jarvik 2000 axial flow device, when the outflow cannula was anastomosed to the descending aorta, it has never been reported so far with ascending aortic anastomosis [5].

The implications of this debate are obvious. If maximal cardiac output and LV decompression are the goal, the aortic valve should stay permanently closed, the interatrial septum should be shifted to the left, and flow profile in the outflow cannula will be less pulsatile. On the other hand, if pulsatility is the objective, intermittent aortic valve opening, neutral or even rightward shift of interatrial septum, and hence more flow pulsatility in the outflow cannula will be expected (Figure 5).

In our institution, the usual parameters used for estimation of proper LVAD function include (1) status of aortic valve opening; (2) interatrial and (to a lesser extent) interventricular septum position; (3) flow pulsatility in the outflow cannula; (4) TR velocity; (5) estimation of right atrial pressure; (6) LVAD system output and total cardiac output estimation.

The aortic valve opens whenever systolic LV pressure increases above the aortic pressure. Any reduction in LVAD function or speed will result in reduced LV unloading, increased LV systolic pressure reaching above aortic systolic pressure, allowing aortic valve opening, and systolic ejection. Increased native LV contractility (as encountered during stress, inotropic support, or even cardiac recovery) and increased preload may result in aortic valve opening. In case of increased afterload, the status of aortic valve opening 

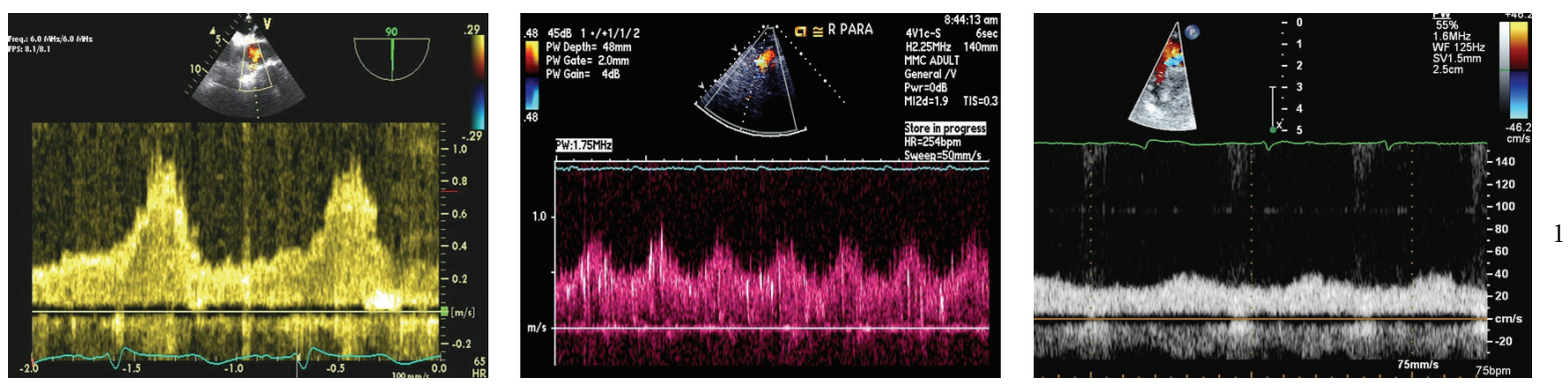

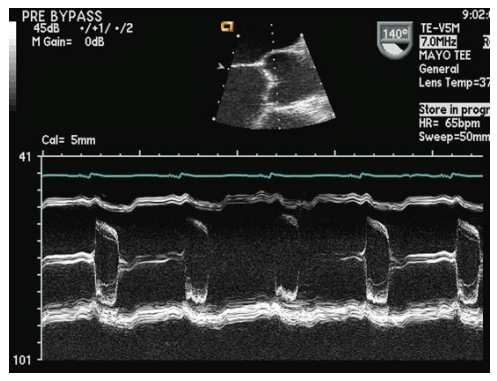

(a)

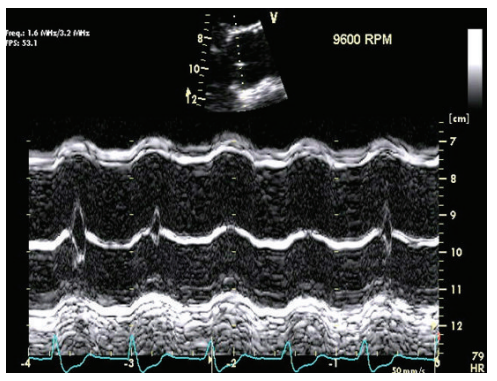

(b)

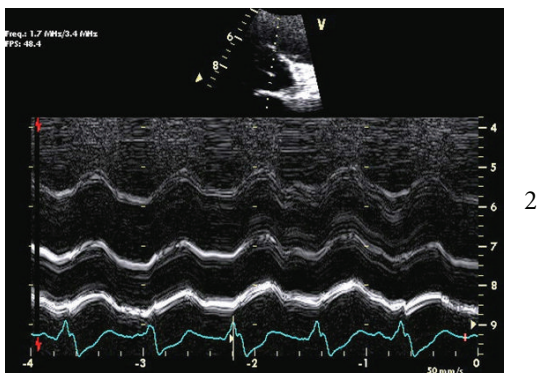

(c)

FIGURE 5: LVAD speed settings. Columns: (a) minimal speed; (b) submaximal speed; (c) maximal speed. Rows: (1) pulsed Doppler, outflow cannula; (2) M mode aortic valve. The status of aortic valve opening and aortic flow pulsatility depends on pump speed and output, LV contractility, preload, and afterload conditions. Reduction in LVAD speed to minimum will result in minimal unloading of the LV, increased LV systolic pressure above aortic pressure allowing pulsatile flow (a1), aortic valve opening during every cycle, seen in M mode (a2). Increasing the speed to submaximal, will unload the ventricle, reduce systolic left ventricular pressure, decrease the outflow pulsatility (b1), and result in intermittent aortic valve opening in $\mathrm{M}$ mode (b2). Increasing speed to maximum will unload the left ventricle even further, resulting in almost continuous aortic flow (c1), and no aortic valve opening (c2).

depends on the site of disturbance. If the reason for increased afterload is outflow cannula obstruction or kinking, the left ventricular systolic pressure will increase, but aortic pressure will decrease resulting in aortic valve opening every cycle. If on the other hand, the increased afterload is due to vasoconstriction, the aortic and left ventricle pressure will increase concomitantly, resulting in a closed aortic valve.

The position of the interatrial septum is the most sensitive measure for estimating proper decompression of left heart filling pressures. Furthermore, contrary to aortic valve opening status, it also shows the efficiency of diastolic decompression. Importantly, it should be done concurrently with estimation of right atrial pressure (using the IVC method) as increased RA pressure may cause leftward deviation of interatrial septum in the presence of high left heart filling pressure.

Pump flow, although it can be extracted from the device controller, must be directly evaluated during TTE examination. We believe that measuring the outflow cannula diameter in the right parasternal view, calculating its surface area, and multiplying it with the outflow cannula flow integral is the most reproducible method. For total cardiac output, our practice is to measure the right ventricular outflow tract (RVOT) diameter in the short axis view on the level of aortic valve and calculate its surface area. The result is multiplied by the integral of flow in the RVOT (on the same view) and the heart rate.
6.2. Assessment of Recurrent Heart Failure Associated with LVAD Dysfunction. When assessing a patient with recurrent heart failure symptoms, an integrated clinical and echocardiographic management protocol is used as a guide to recognize the cause of axial pump failure [25]. The patient is connected to the system monitor displaying a variety of system performance data including pump speed, power, pulsatility index (PI), and pump flow. Briefly, the pump speed will be determined during a speed ramp study. Pump power is a direct measurement of motor voltage and current. Increases in pump speed, flow, or physiological demand will increase pump power. Specifically, gradual power increases, power values greater than 10-12 watts, or abrupt changes in power should raise concern for possible thrombus inside the pump. When the LV contracts, the increase in ventricular pressure causes an increase in pump flow during systole. The magnitude of these flow changes is measured to produce the PI. The PI represents cardiac pulsatility and is related to the magnitude of assistance provided by the LVAD. Higher values indicate more ventricular filling or better contractility (pump is providing less support to the LV), while lower values indicate less ventricular filling or lower contractility. Pump flow is estimated based on power. Since it is a calculated value, it becomes imprecise at low and high regions of the power-flow relationship. Therefore, any increase in power not related to an increased flow, such as thrombus, will cause an erroneously high flow. Conversely, an occlusion of flow 
path (inflow obstruction due to malposition or suction events) will decrease power and calculated flow. In either situation, an independent assessment of pump output using the TTE should be performed. It is however important to mention that no single monitor parameter is an adequate surrogate for monitoring the clinical status of the patient.

6.3. Evaluation of LVAD Dysfunction. LVAD malfunctions can be broadly categorized into three main groups: (1) low echocardiographically estimated pump flow and increased power values; (2) low echocardiographically estimated pump flow with normal or low power values; (3) high echocardiographically estimated pump flow with low echocardiographically estimated forward cardiac output.

6.3.1. Low Pump Flow with Increased Power Values. The reasons for this combination are pump failure or increased afterload. Pump failure may result from thrombosis, mechanical malfunction, or decreased speed settings. The echocardiographic appearance of these etiologies includes some or all of the following: (1) rightward deviation of the interventricular septum; (2) significant functional mitral regurgitation; (3) aortic valve opening every cardiac cycle; (4) spontaneous echo contrast in the left atrium or left ventricle; (5) regurgitant flow through the inflow and outflow cannula, for reasons previously discussed. Reduced speed settings will be obvious from the controler device interrogation, while presence of left ventricular or left atrial thrombus and increased power and controler calculated flow should raise suspicion for LVAD thrombosis. This differential diagnosis is clinically crucial, as LVAD thrombosis may be managed by anticoagulants or thrombolysis, while LVAD malfunction may require urgent surgery [25]. On the other hand, increased afterload differential diagnosis includes outflow cannula obstruction, outflow cannula kinking, and extreme systemic vasoconstriction [25]. Outflow cannula kinking should be suspected when there is loss of Doppler signal in the outflow cannula in any echocardiographic view $[23,25]$. This can be confirmed by catheterization and contrast injection [26]. In the situation of severe systemic vasoconstriction, one should bare in mind that axial LVADs are extremely sensitive to increased afterload, resulting in decline in pump output. Differentiation of systemic vasoconstriction from mechanical complication is essential as vasoconstriction will be treated conservatively. Although most of the echocardiographic criteria for LVAD dysfunction will be present in patients with severe vasoconstriction, the aortic valve will stay closed. PW Doppler interrogation of the outflow cannula will demonstrate high velocity pulsatile forward flow, and PI will be increased [25]. The reason for this set of findings is that although LVAD output is reduced as demonstrated by the septal shift and functional $\mathrm{MR}$, the impeller is still rotating, amplifying the pressure wave received from the LV. Under conditions of low pump flow, the pressure in the left ventricle is higher than normal, resulting in increased forward velocity in the outflow cannula. Although the pressure in the LV is higher than normal, the pressure in the aorta is even higher, preventing aortic valve opening and systolic ejection.
6.3.2. Low Pump Flow with Normal Current and Power Values. The combination of low pump flow with normal or low power (and low PI) values is the result of reduced LVAD preload. Reduced preload is most commonly encountered with RV failure, significant TR, or hypovolemia. Another common reason for reduced LVAD preload is inflow cannula obstruction, largely due to malposition and intermittent obstruction by the adjacent LV walls. The apical cannula should stay central, not abutting any wall. Color, CW, and PW Doppler interrogation should be done as already described. Inflow cannula obstruction will result in high velocity aliased flow at the orifice with manifest convergence area. Other rare reasons for reduced LVAD preload include mitral stenosis and ventricular fibrillation [27], which can present as recurrent heart failure episodes due to loss of RV function, resulting in reduced LVAD preload and output.

6.3.3. High Pump Flow with Low Forward Cardiac Output. Whenever the calculated total cardiac output is lower than the LVAD output, futile cycles should be suspected. The most common malfunction of the older pulsatile LVAD was inflow valve regurgitation, resulting in extremely high LVAD output, with markedly reduced total cardiac output. This combination of findings can also be encountered in patients with severe AI.

\section{Conclusion}

Precise transthoracic echocardiographic monitoring is mandatory and paramount to evaluate the performance of continuous flow left ventricular assist devices. This evaluation is essential for surgical planning and interventional success. Standard TTE techniques allow optimal LVAD settings during routine follow-up visits and rapid and accurate evaluation of mechanical or systemic malfunctions.

\section{Conflict of Interest}

The authors have no financial associations or relationship with an industry that might pose a conflict of interest with the submitted article.

\section{References}

[1] S. J. Park, A. Tector, W. Piccioni et al., "Left ventricular assist devices as destination therapy: a new look at survival," Journal of Thoracic and Cardiovascular Surgery, vol. 129, no. 1, pp. 9$17,2005$.

[2] O. H. Frazier, E. A. Rose, M. C. Dz et al., "Multicenter clinical evaluation of the HeartMate vented electric left ventricular assist system in patients awaiting heart transplantation," Journal of Thoracic and Cardiovascular Surgery, vol. 122, no. 6, pp. 1186-1195, 2001.

[3] O. H. Frazier, E. A. Rose, M. C. Dz et al., "Multicenter clinical evaluation of the heartmate; vented electric left ventricular assist system in patients awaiting heart transplantation," The Journal of Heart and Lung Transplantation, vol. 20, no. 2, pp. 201-202, 2001.

[4] E. A. Rose, A. C. Gelijns, A. J. Moskowitz et al., "Long-term use of a left ventricular assist device for end-stage heart failure," 
New England Journal of Medicine, vol. 345, no. 20, pp. 14351443, 2001.

[5] O. H. Frazier, T. J. Myers, S. Westaby, and I. D. Gregoric, "Clinical experience with an implantable, intracardiac, continuous flow circulatory support device: physiologic implications and their relationship to patient selection," Annals of Thoracic Surgery, vol. 77, no. 1, pp. 133-142, 2004.

[6] B. P. Griffith, R. L. Kormos, H. S. Borovetz et al., "HeartMate II left ventricular assist system: from concept to first clinical use," Annals of Thoracic Surgery, vol. 71, no. 3, pp. S116-S120, 2001.

[7] O. H. Frazier, R. M. Delgado, B. Kar, V. Patel, I. D. Gregoric, and T. J. Myers, "First clinical use of the redesigned HeartMate II left ventricular assist system in the United States: a case report," Texas Heart Institute Journal, vol. 31, no. 2, pp. 157159, 2004.

[8] L. W. Miller, F. D. Pagani, S. D. Russell et al., "Use of a continuous-flow device in patients awaiting heart transplantation," New England Journal of Medicine, vol. 357, no. 9, pp. 885-896, 2007.

[9] M. P. Siegenthaler, S. Westaby, O. H. Frazier et al., "Advanced heart failure: feasibility study of long-term continuous axial flow pump support," European Heart Journal, vol. 26, no. 10, pp. 1031-1038, 2005.

[10] H. A. Khalil, W. E. Cohn, R. W. Metcalfe, and O. H. Frazier, "Preload sensitivity of the jarvik 2000 and HeartMate II left ventricular assist devices," ASAIO Journal, vol. 54, no. 3, pp. 245-248, 2008.

[11] N. C. Dang, V. K. Topkara, M. Mercando et al., "Right heart failure after left ventricular assist device implantation in patients with chronic congestive heart failure," Journal of Heart and Lung Transplantation, vol. 25, no. 1, pp. 1-6, 2006.

[12] N. D. Patel, E. S. Weiss, J. Schaffer et al., "Right heart dysfunction after left ventricular assist device implantation: a comparison of the pulsatile HeartMate I and axial-flow HeartMate II devices," Annals of Thoracic Surgery, vol. 86, no. 3, pp. 832-840, 2008.

[13] F. Waldenberger, Y. I. Kim, S. Laycock, B. Meyns, and W. Flameng, "Effects of failure of the right side of the heart and increased pulmonary resistance on mechanical circulatory support with use of the miniaturized HIA-VAD displacement pump system," Journal of Thoracic and Cardiovascular Surgery, vol. 112, no. 2, pp. 484-493, 1996.

[14] B. Pinamonti, M. Zecchin, A. Di Lenarda, D. Gregori, G. Sinagra, and F. Camerini, "Persistence of restrictive left ventricular filling pattern in dilated cardiomyopathy: an ominous prognostic sign," Journal of the American College of Cardiology, vol. 29, no. 3, pp. 604-612, 1997.

[15] S. F. Yiu, M. Enriquez-Sarano, C. Tribouilloy, J. B. Seward, and A. J. Tajik, "Determinants of the degree of functional mitral regurgitation in patients with systolic left ventricular dysfunction: a quantitative clinical study," Circulation, vol. 102, no. 12, pp. 1400-1406, 2000.

[16] G. M. Scalia, P. M. McCarthy, R. M. Savage, N. G. Smedira, and J. D. Thomas, "Clinical utility of echocardiography in the management of implantable ventricular assist devices," Journal of the American Society of Echocardiography, vol. 13, no. 8, pp. 754-763, 2000.

[17] W. A. Zoghbi, M. Enriquez-Sarano, E. Foster et al., "Recommendations for evaluation of the severity of native valvular regurgitation with two-dimensional and Doppler echocardiography," Journal of the American Society of Echocardiography, vol. 16 , no. 7, pp. 777-802, 2003.
[18] S. Chumnanvej, M. J. Wood, T. E. MacGillivray, and M. F. V. Melo, "Perioperative echocardiographic examination for ventricular assist device implantation," Anesthesia and Analgesia, vol. 105, no. 3, pp. 583-601, 2007.

[19] S. J. Park, K. K. Liao, R. Segurola, K. P. Madhu, and L. W. Miller, "Management of aortic insufficiency in patients with left ventricular assist devices: a simple coaptation stitch method (Park's stitch)," Journal of Thoracic and Cardiovascular Surgery, vol. 127, no. 1, pp. 264-266, 2004.

[20] S. C. Horton, R. Khodaverdian, P. Chatelain et al., "Left ventricular assist device malfunction: an approach to diagnosis by echocardiography," Journal of the American College of Cardiology, vol. 45, no. 9, pp. 1435-1440, 2005.

[21] B. J. Kircher, R. B. Himelman, and N. B. Schiller, "Noninvasive estimation of right atrial pressure from the inspiratory collapse of the inferior vena cava," American Journal of Cardiology, vol. 66, no. 4, pp. 493-496, 1990.

[22] J. Ferns, R. Dowling, and G. Bhat, "Evaluation of a patient with left ventricular assist device dysfunction," ASAIO Journal, vol. 47, no. 6, pp. 696-698, 2001.

[23] E. Catena, F. Milazzo, M. Merli et al., "Echocardiographic evaluation of patients receiving a new left ventricular assist device: the Impella $($ recover 100," European Journal of Echocardiography, vol. 5, no. 6, pp. 430-437, 2004.

[24] K. D. May-Newman, B. K. Hillen, C. S. Sironda, and W. Dembitsky, "Effect of LVAD outflow conduit insertion angle on flow through the native aorta," Journal of Medical Engineering and Technology, vol. 28, no. 3, pp. 105-109, 2004.

[25] E. Catena, F. Milazzo, E. Montorsi et al., "Left ventricular support by axial flow pump: the echocardiographic approach to device malfunction," Journal of the American Society of Echocardiography, vol. 18, no. 12, pp. 1422.e7-1422.e13, 2005.

[26] S. C. Horton, R. Khodaverdian, A. Powers et al., "Left ventricular assist device malfunction: a systematic approach to diagnosis," Journal of the American College of Cardiology, vol. 43, no. 9, pp. 1574-1583, 2004.

[27] M. Bedi, R. Kormos, S. Winowich, D. M. McNamara, M. A. Mathier, and S. Murali, "Ventricular arrhythmias during left ventricular assist device support," American Journal of Cardiology, vol. 99, no. 8, pp. 1151-1153, 2007. 


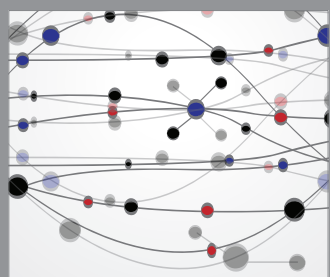

The Scientific World Journal
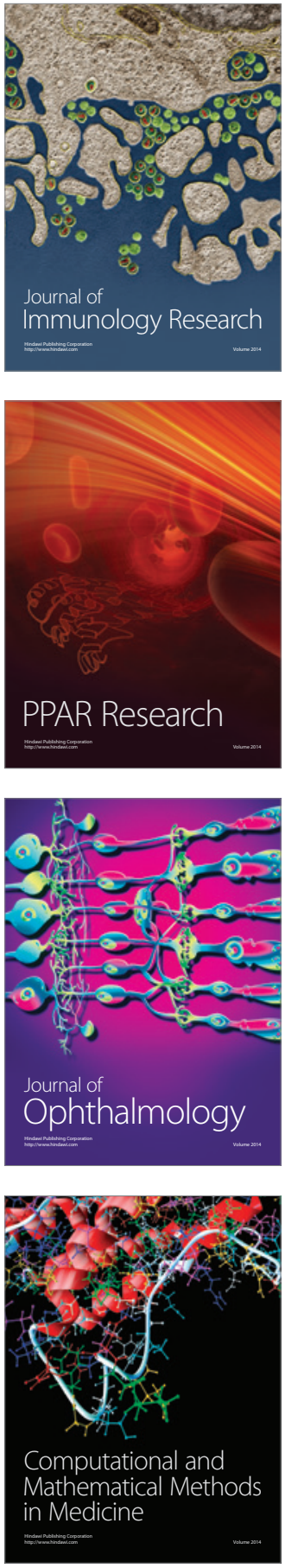

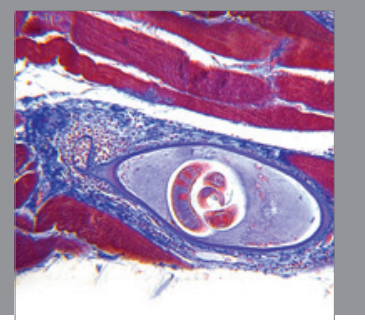

Gastroenterology

Research and Practice
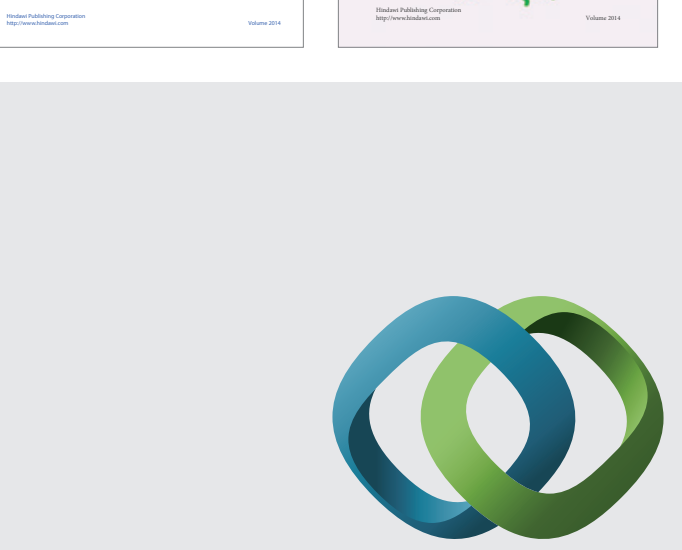

\section{Hindawi}

Submit your manuscripts at

http://www.hindawi.com
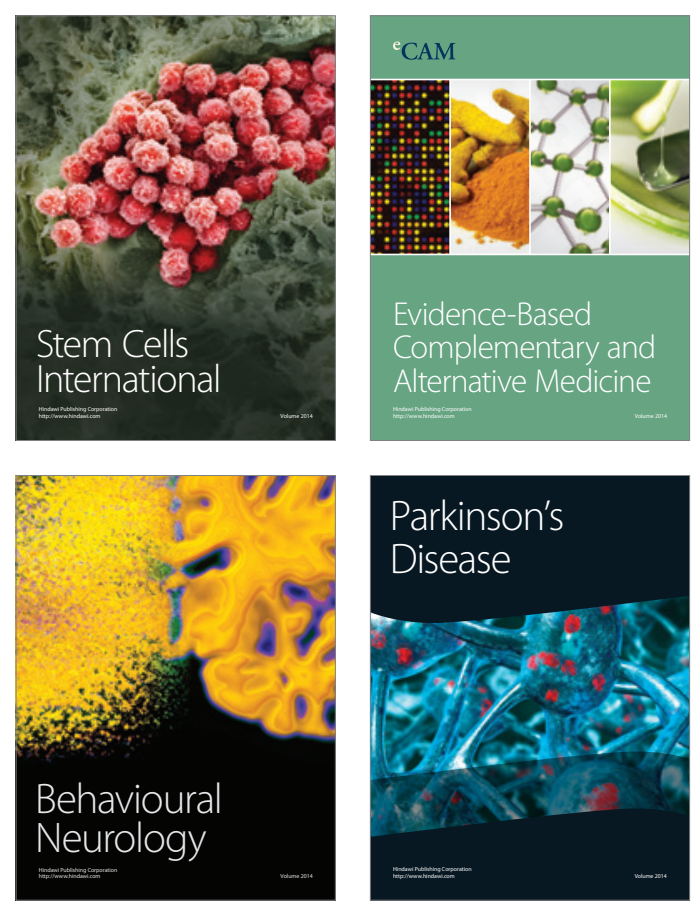

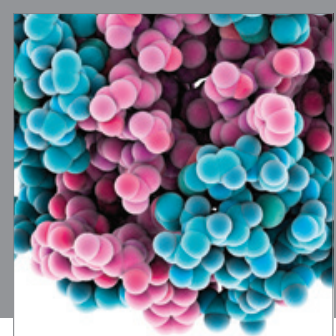

Journal of
Diabetes Research

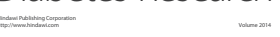

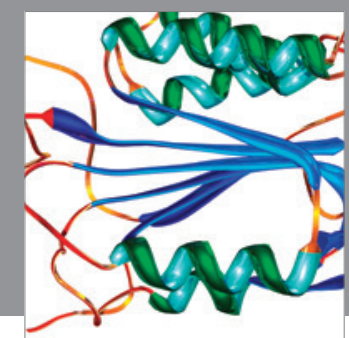

Disease Markers
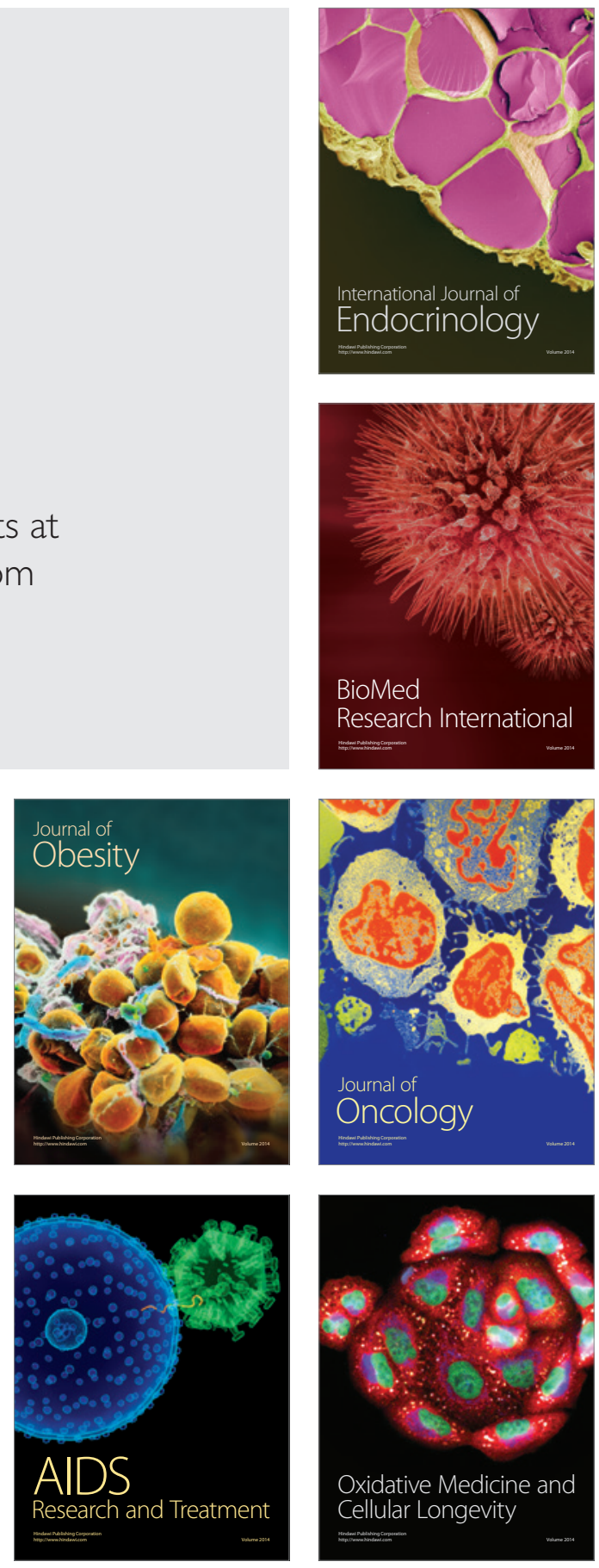